\title{
A hybrid global MPPT scheme based on power closed-loop scanning and $\mathrm{P} \& \mathrm{O}$ method
}

\author{
Li Shanshou ${ }^{\mathrm{a}, \mathrm{b}}$, Zhang Xing ${ }^{\mathrm{a}}$, Gu Jun ${ }^{\mathrm{a} *}$ \\ ${ }^{a}$ HeFei University of Technology, No.193 of Tunxi Road, Hefei 230009, China \\ ${ }^{b}$ Anhui Jianzhu University, No.292 of Ziyun Road, Hefei 230601, China
}

\begin{abstract}
This paper presents a hybrid Global Maximum Power Point Tracking (GMPPT) method. Firstly, an improved global scanning method is presented, which overcomes the difficulty to determine the power step size in "power closed-loop scanning (PCS)". Secondly, an improved P\&O method is employed as the steady-state tracking method, in which some improvement measures was proposed for eliminating the problem of the steady-state oscillation and the dynamic misjudging. Thirdly, a novel restarting strategy is proposed, which happens with a novel criterion for judging the environment change. At last, some comparative experiments with other GMPPT algorithms are presented to highlight the significance of the presented approach.
\end{abstract}

Keywords: Partial shading, Global Maximum Power Point Tracking (GMPPT), Power Closed-Loop Scanning (PCS), Perturb and Observe (P\&O) method

\section{Introduction}

When PV array operates under partial-shading conditions, the power-voltage characteristic curve exhibits multiple Maximum Power Points (MPP) [1]. Under such case, the conventional Maximum Power Point Tracking(MPPT) techniques [2] designed for single MPP case, may be getting into a local MPP and fail to track the global MPP, which results in significant reduction of the generated power and the overall efficiency of system.

In order to alleviate the partial-shading effects, different array configurations(e.g. SP, TCT, and BL) was tested [3], the comparative study shows that BL and TCT configurations result in a $2.3 \%$ and $3.8 \%$ increase in MPP compared with the SP configuration. Another approach is the use of distributed electronics in PV systems which was called for distributed MPPT (DMPPT) technique [4] or power optimizer [5]. The biggest problem within these methods is the cost increase significantly, and the system is more difficult to control. An economic and effective measure for improving the efficiency of PV systems suffering from partial-shading problem is the application of the Global Maximum Power Point Tracking (GMPPT) algorithm.

Over the past few decades, the research on GMPPT method has become a hot topic in PV system, and many GMPPT algorithms have been proposed [6]. In Ref. [7] a two stage method based on the reference impedance $\left(R_{r e f}\right)$ tracking and perturb-and-observe method is proposed, the main shortage is that the reference impedance must be calculated by measuring the short-circuit current $\left(I_{s c}\right)$ and the open-circuit voltage $\left(U_{o c}\right)$. In Ref. [8], [9], two improved GMPPT methods are proposed in order to avoid the shortage of two stage method. The main disadvantages of these method is the dependence on the information of PV array structure and the possibility of falling into the local MPP. In Ref. [10], [11], an innovative global scanning scheme named "Power Closed-Loop Scanning (PCS)" is proposed, which does not rely

\footnotetext{
* Manuscript received May 25, 2015; revised September 15, 2015.

Corresponding author. Tel.: +86-551-6382-8177; E-mail address:xlisq79@163.com.

doi: $10.12720 /$ sgce.4.4.291-298
} 
on any knowledge of the electrical characteristics of PV modules or their configuration within PV array. However, the obvious shortage is the dilemma in weighting the accuracy and speed of scanning process and determining the power step size. In Ref. [12]-[14], some GMPPT methods based on heuristic intelligent search algorithm (e.g. PSO algorithm) are presented, which can track the global MPP with a higher success rate in the static environment and eliminate the oscillation problem inherent in P\&O method. However, the shortage of these methods is also obvious, it is unreliable to fulfill GMPPT in dynamic environment, because the memorial information in PSO algorithm is not always valid in these situations. Moreover, the complexity of PSO algorithm is also an obstacle in practical applications.

In this paper, a hybrid GMPPT scheme is proposed, which consists of three main parts. The first part is an effective global scanning method. The second component is a steady-state tracking strategy, which is essentially an improved $\mathrm{P} \& \mathrm{O}$ method. The third part is a relatively perfect dynamic environment judging criterion and the restarting mechanism. The rest of this paper is arranged as follows: In Section II, the global scanning method will be analyzed. In Section III and IV, The steady-state tracking strategy and the dynamic environment judging criterion and the restarting mechanism will be described comprehensively. In Section V, the feasibility and effectiveness of the method is verified by a set of contrast experiments. Finally, the paper is concluded in Section VI.

\section{The Global Scanning Method}

\subsection{Shortages in power closed-loop scanning [10] [11]}

Traditionally, the output of MPPT algorithm is the reference voltage $\left(U_{r e f}\right)$, which is modified in every MPPT period $\left(T_{m p p t}\right)$. The control program calculate the control signal $(d)$ in each control period $\left(T_{c}\right)$, according to the error of $\left(U_{\text {ref }}\right)$ and the output voltage $\left(U_{p v}\right)$ of PV array. as shown in (1). Where the symbol $\left(f_{u}\right)$ is the voltage control function (e.g. PI ).

$$
d=f_{u}\left(U_{r e f}-U_{p v}\right)
$$

As described in Ref. [10], [11], the output of MPPT algorithm with PCS, is the reference power $\left(P_{\text {ref }}\right)$ instead of $U_{\text {ref }}$. The control program generate the control signal ( $d$ ) in each control period according to the power error of $\left(P_{r e f}\right)$ and the real-time power $\left(P_{p v}\right)$ of PV array. This process is as (2). Where the $\operatorname{symbol}\left(f_{p}\right)$ is the power control function for (e.g. PI ).

$$
d=f_{u}\left(f_{p}\left(P_{r e f}-U_{p v} * I_{p v}\right)-U_{p v}\right)
$$

The process of PCS can be described as follows. GMPPT algorithm increase $P_{\text {ref }}$ with the power step size $\left(P_{\text {step }}\right)$ in each MPPT period. If $P_{r e f}$ is less than the global maximum power, the system will sequentially stabilize to the point where $P_{p v}$ is equal to $P_{r e f}$. If $P_{r e f}$ is greater than the global maximum power, the voltage of PV array will quickly slide to the left side and eventually stabilizes to the lowest boundary voltage $\left(U_{\min }\right)$. Thereafter, the system enters the steady-state tracking. The advantage of PCS is that which can get across the valley area between MPPs in one $T_{m p p t}$, which result in a quickly global scanning speed and achieve the global MPP positioning. The disadvantage of PCS is that the scanning speed and positioning accuracy cannot be targeted at the same time, PCS may fail to accurately locate the global MPP with a larger power step size. If the power step size is reduced, the positioning accuracy can be improved, but it will lead to the longer scanning process..

\subsection{The improved global scanning strategy}

As everyone knows, the biggest problem in the traditional constant voltage step-size scanning (CVS) process is that which cannot judge the valley area on a multiple MPP curve, but it has a higher scanning accuracy. Combining PCS with CVS can form an improved global scanning scheme, which can overcome shortcomings of them and achieve a balance between the accuracy of positioning and the speed of 
scanning. The proposed scheme is shown in Fig. 1. The detailed description of the improved scanning scheme is as follows: In each $T_{m p p t}$, if the current power $(P[n])$ is greater than the power $(P[n-1])$ of the previous $T_{m p p t}$, the variable "Var" will be set with "U", the output MPPT algorithm is $U_{\text {ref }}$, such as points "a i" in Fig. 1. The control program calculate the control signal according to (1) until the next $T_{m p p t}$. Otherwise, if $P[n]$ is less than $P[n-1]$, the variable "Var" will be set with "P", the MPPT algorithm output $P_{r e f}$, such as point "i j" in Fig. 1. The control program compute the control signal according to (2) until the next $T_{m p p t}$. When $U_{p v}$ reachs $U_{m i n}$, the global search process will be terminated. The MPPT algorithm will enter the steady-state tracking. The status indicator variable "Stage" is assigned to 2, the variable "Var" is set with " $\mathrm{U}$ " and $U_{r e f}$ is set equal to $U[n-1]$, The flowchart of the proposed global scanning scheme is shown in Fig. 2 (Stage 1).

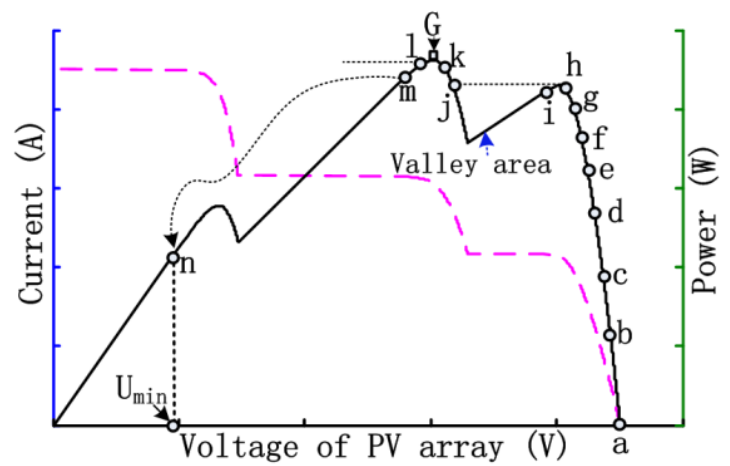

Fig. 1. Sketch map of the improved scanning scheme.

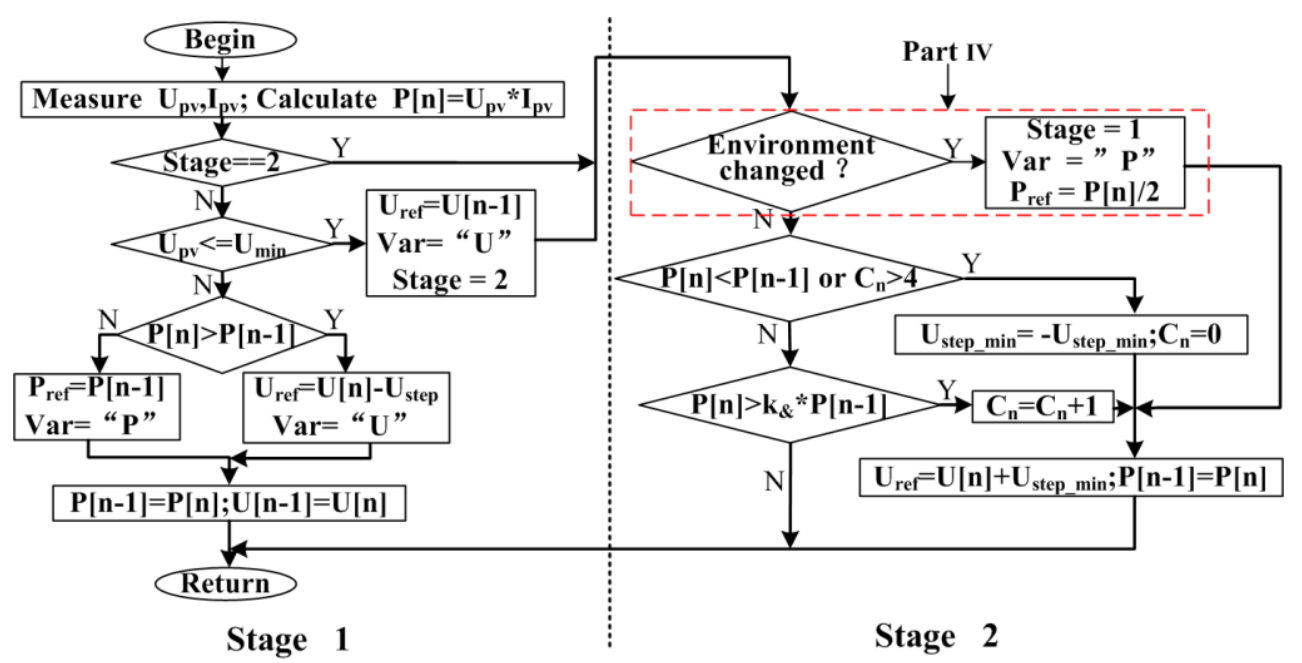

Fig. 2. Flowchart of the proposed GMPPT scheme.

\section{Tracking Method in Steady-State}

\subsection{The deficiency of $P \& O$ method}

The P\&O method, which has many advantages (e.g. simplicity, easy to implement), is widely used in the single peak MPPT procedure. However, it has the defect of misjudging under dynamic environment and the oscillation in steady-state [2]. In the traditional P\&O method, As long as the judging criteria $(P[n]>=P[n-1])$ is met, the algorithm will keep the previous perturbation direction. When the irradiance increases continuously under dynamic circumstance and the judging criteria is always met, which will lead to misjudging phenomenon. If the misjudging occurred, the working point will move in 
one direction and go far away from the global MPP. In steady-state, even though there is only a small increment $\left(\delta_{P}\right)$ between $P[n]$ and $P[n-1]$, which may be induced by environmental disturbance, the $\mathrm{P} \& \mathrm{O}$ algorithm will maintain the previous direction of perturbation until the criteria $(P[n]<P[n-1])$ is met, which leads to the steady-state oscillation in a three-point or four-point mode [2].

\subsection{The improved $P \& O$ method}

According to the above analysis, the judging criterion which come to the conclusion of maintaining the previous perturbation direction is not perfect. In order to overcome defects of the traditional $\mathrm{P} \& \mathrm{O}$ method, the judging criterion must be modified. The flowchart of the improved P\&O method is shown in Fig. 2 (Stage 2). The first improvement is that a counter $\left(C_{n}\right)$ is used to count the step number of single direction continuous movement, when the condition $\left(C_{n}>4\right)$ is met, the direction of movement will be forcibly changed, which can confirm the validity of a continuously moving and avoid the misjudging under dynamic circumstance. The second improvement is the judging criterion which comes to the conclusion of maintaining the previous perturbation direction is modified, that is $\left(P[n]>=k_{\&} * P[n-1]\right)$, the symbol $\left(k_{\&}=1.01\right)$ is a constant in this paper. According to the improved criterion, the system stays at the present point if $\left(0<=k_{\&}<=1.01\right)$, the information of $(U[n], P[n-1])$ and $\left(U_{\text {ref }}\right)$ will not be updated until the criteria $\left(P[n]>=k_{\&} * P[n-1]\right)$ or $(P[n]<P[n-1])$ is meet, so far, the oscillations in the steady-state of $\mathrm{P} \& \mathrm{O}$ can be effectively reduced. In steady-state the step size of the $\mathrm{P} \& \mathrm{O}$ method is decreased to " $U_{\text {step_min }}$ ", $\left(U_{\text {step_min }}=2 V\right.$ in this paper $)$.

\section{Judging Criterion for Dynamic Environment and Restarting Mechanism}

\subsection{Judging criterion for dynamic environment}

MPPT algorithm should restart if the environment changed. In this process, the first issue is to determine the environment change. A perfect criteria for judging the dynamic environment can reduce the restarting number of MPPT algorithm and improve the overall efficiency of the PV system. At present, most GMPPT method is mainly according to the change of the output power to judge the environment changes [8]-[11]. The typical criterion are shown in formula (3).

$$
\left\{\begin{array}{l}
\left|\frac{P[n]-P[n-1]}{P[n-1]}\right|>K_{\text {set }} \\
|\Delta U[n]|=|U[n]-U[n-1]| \leq \Delta U_{\text {set }}
\end{array}\right.
$$

In (3), the constant ( $K_{\text {set }}$ ) is the threshold of the power change (generally $K_{\text {set }}=0.1$ ) [8], the symbol ( $\Delta U_{\text {set }}=2 \mathrm{~V}$ in this paper) is the setting for the voltage threshold in steady state. The main shortcoming of the judging criteria is that the slowly changing process of environment can't be judged and the changing under single MPP case will lead to unnecessary restarting.

In order to overcome these flaws, this paper introduces the cumulative change of the output power and the output voltage in steady-state into the judging criterion, as shown in the formula (4).

$$
\left\{\begin{array}{l}
|U[n]-U[n-1]|<\Delta U_{\text {set }} \\
\sum(U[n]-U[n-1]) \geq U_{\text {set }} \\
{\left[\sum(P[n]-P[n-1])\right] / P[n] \geq K_{\text {set }}}
\end{array}\right.
$$

In (4), the symbol ( $U_{\text {set }}=10 \mathrm{~V}$ in this paper) is the voltage threshold for distinguishing the multiple MPP case and the single MPP case. Under the single MPP case, the cumulative change of voltage is very small when irradiance fluctuate. Under the multiple MPP case, the cumulative change of the output power will gradually increase and the working point will move significantly. Considering the cumulative change of the output power and the output voltage at the same time can effectively judge the environmental 
gradient changing process and reduce the unnecessary restarting number.

\subsection{Restarting mechanism of MPPT method}

When the environment changes, MPPT algorithm should be restart and reenter steady-state in a stable and fast way. But it is difficult to achieve with the conventional scheme which restart MPPT algorithm based on the voltage of PV array [8], [9], because the voltage of the PV array is affected significantly by the temperature and irradiance, which mean that the information of the memorial voltage is no longer valid when restart. The system must be restarted from the open-circuit state, this will bring to the unnecessary power loss.

In order to overcome these deficiencies, PCS can be introduced into the restarting process, in which the MPPT algorithm based on the current output power $(P[n])$ sets the reference power $\left(P_{\text {ref }}\right)$ of restarting point. Taking into account the probability of that PV array is shaded over half of the area is low, the reference power $\left(P_{r e f}\right)$ can be given as (5). After that the control program perform "power closed-loop control" and give the reference voltage of restarting.

$$
P_{r e f}=P[n] / 2
$$

Fig. 3 shows the process of judging dynamic environment and restarting MPPT algorithm based on PCS. Suppose that the working point is initially at point "M", after the environment change the working point move to point "a" , after that the working point will gradually move to point "b" with $\mathrm{P} \& \mathrm{O}$ algorithms. At this point the accumulated change of the voltage and power reaches the threshold in formula (4) , and MPPT algorithm will restart after setting the variable "Stage $=1$ " and the variable "Var $=\mathrm{P}$ " as shown in Fig. 3. In the next MPPT period the working point move to point "c" based on PCS. In the second MPPT period, because $P[n]$ standing for the power at point "c" is lower $P[n-1]$ standing for the power at point "b", MPPT algorithm output the reference power $\left(P_{r e f}=P[n-1]\right)$ because the criteria $(P[n]<P[n-1])$ is met according to the improved global scanning method, the working point transfer to point " $b$ " " based on PCS.

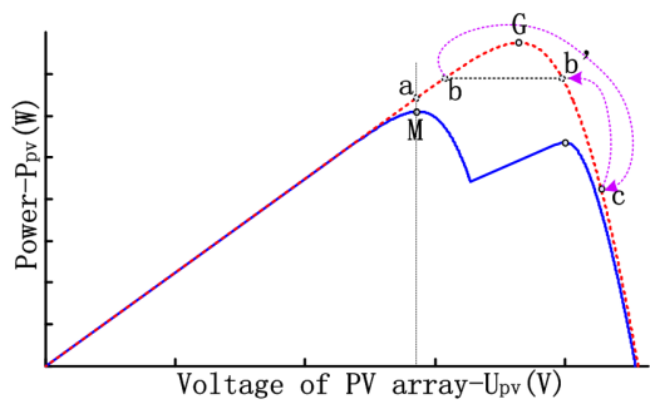

Fig. 3. Diagram of the restarting process based on PCS.

\section{Experiment Verification and Analysis}

\subsection{Experiment conditions}

Experiment conditions are the same as [10]. PV array is simulated by CHROMA PV simulator. The system adopts a two stage structure, and the MPPT function is realized by the former BOOST circuit. The control period is 0.0002 second $\left(T_{c}=0.0002 s\right)$, the MPPT period is 1 second $\left(T_{m p p t}=1 s\right)$. The MPPT algorithm and the control program is implemented with DSP-TMS320F28335.

The contrasted methods are the POC method [8], [9] and the PSO algorithm [12]-[14]. Parameters in the proposed method and POC method: Initial step ( $\left.U_{\text {step }}\right)$ is $8 \mathrm{~V}$, and the minimum step size ( $\left.U_{\text {step_min }}\right)$ is $2 \mathrm{~V}$; Parameters in PSO method [12]-[14]: $w=0.4, C 1=0.2, C 2=2, r l=r 2=0.5$, the maximum particle velocity is $15 \mathrm{~V}$, Initial positions of four particle respectively is $0.95^{*} \mathrm{Uoc}, 0.75^{*} \mathrm{Uoc}, 0.55^{*} \mathrm{Uoc}, 0.35^{*} \mathrm{Uoc}$. 


\subsection{Static multiple MPP case tests}

The parameters and the P-U curves of the testing PV array are shown in Fig. 4 (a), the global MPP is the left MPP. The waveforms of the output voltage and current with three methods are shown in Fig. 4 (b) (c), (d).

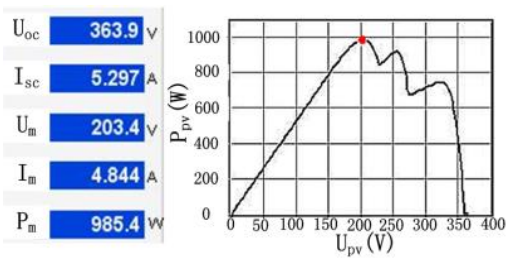

(a)

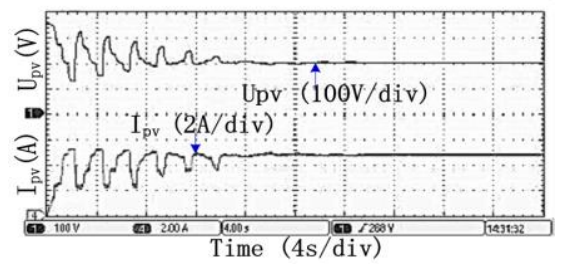

(c)

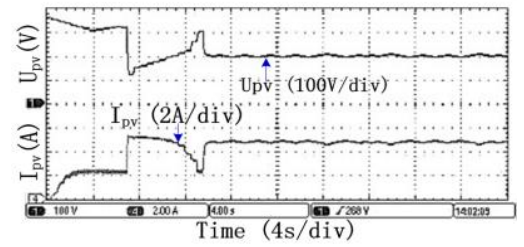

(b)

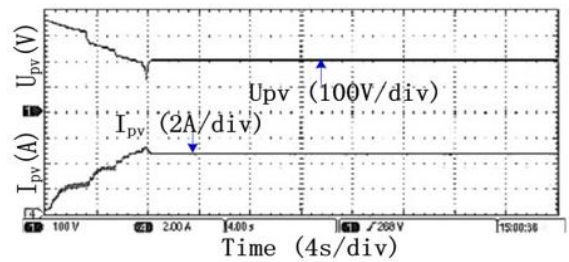

(d)

Fig. 4. Waveforms of MPPT testing under static multiple MPP case (a) P-U curve of testing case (b) Upv and Ipv with POC method (c) Upv and Ipv with PSO method (d) Upv and Ipv with the proposed method.

As shown in Fig. 4 (b) (c) (d), the output voltage and the current is around 203V and 4.8A, three methods are all tracked to the global MPP. But the POC method and the PSO algorithm need 14 seconds for entering the steady-state, the proposed method takes only 8 seconds. Furthermore, the curves of output voltage and current with the POC method has certain amplitude fluctuations as shown in Fig. 4 (b), the curves with the PSO algorithm and the proposed method is without fluctuations. The experimental results indicate that the proposed method has a faster speed and eliminates the oscillation problem of the P\&O method

\subsection{Dynamic tests under single MPP case}

Single MPP case changing continuously, as shown in Fig. 5(a), is an important items of European MPPT testing standard --EN50530. The irradiance reciprocate changes between 100\% and 30\%. Fig. 5(b), (c), (d) are waveforms of the output voltage and current standing for the tracking process with the contrasting methods .

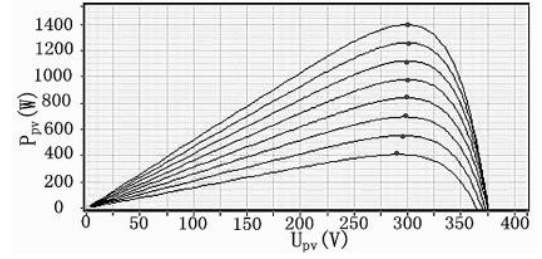

(a)

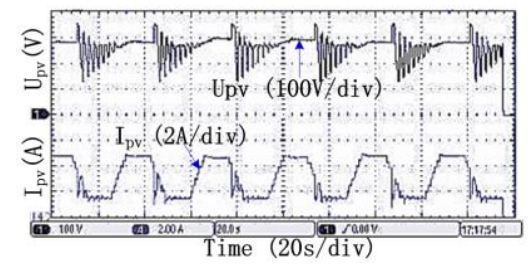

(c)

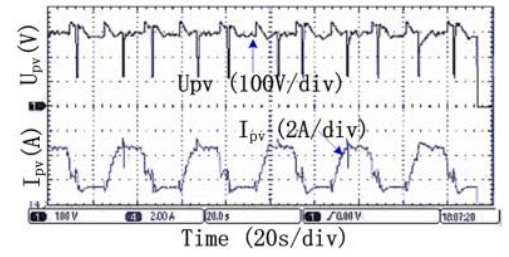

(b)

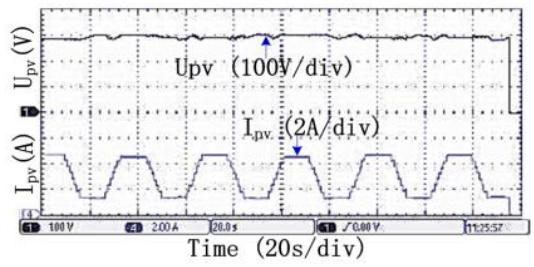

(d)

Fig. 5. Waveforms of MPPT testing under dynamic single MPP case (a) P-U curve of testing case (b) Upv and Ipv with POC method (c) Upv and Ipv with PSO method ( d ) Upv and Ipv with the proposed method. 
As shown in Fig. 5 (b) (c), the POC algorithm and the PSO algorithm restart frequently in the irradiance changing process, because the criterion for judging the dynamic circumstance is only based on the power difference of the current power $(P[n])$ with the previous power $(P[n-1])$ or the maximum power $\left(P_{\max }\right)$ in memory[10]. Nevertheless, the proposed algorithm has not occurred the frequent restarting problem as shown in Fig. 5 (d), because the accumulated change of voltage and power are used as the judging criterion in the proposed method.

The efficiency reports given by CHROMA PV simulator show that the dynamic efficiency with the POC method is between $94.81 \%$ and $95.43 \%$, the efficiency of the PSO algorithm is only $93.29 \%$, the dynamic tracking efficiency of the proposed method is stability between $99.32 \%$ and $99.54 \%$. The results prove that the improved judging criterion can effectively reduce the restarting frequency and improve the dynamic tracking efficiency

\subsection{Dynamic tests under multiple MPP case}

The testing case is that two modules in PV array is shaded, the irradiance of shaded modules gradually decrease from $900 \mathrm{w} / \mathrm{m}^{2}$ to $450 \mathrm{~W} / \mathrm{m}^{2}$ within 120 seconds. The left curve in Fig. 6 (a) is the initial state, and the right one is the final state, between them there are six transitional state.

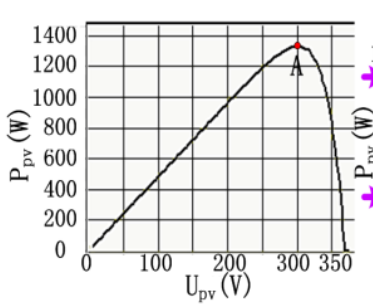

(a)

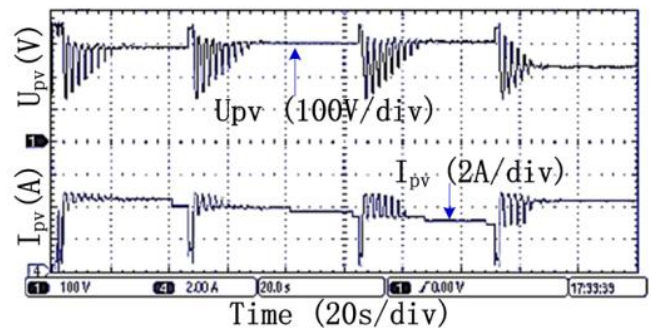

(c)

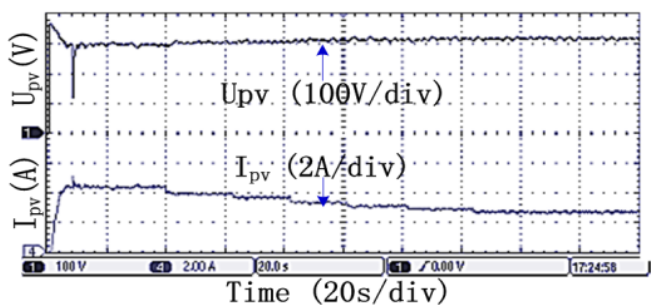

(b)

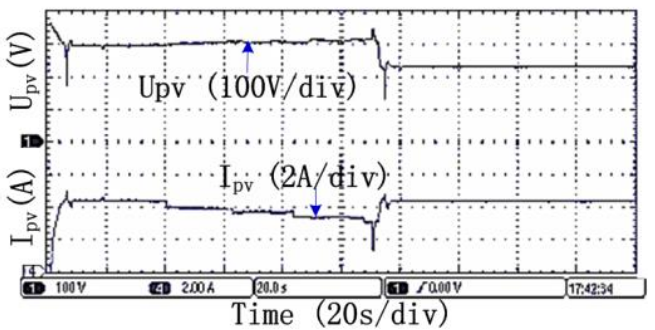

(d)

Fig. 6. Waveforms of MPPT testing under dynamic partial shading case(a) P-U curve of testing case (b) Upv and Ipv with POC method (c) Upv and Ipv with PSO method (d) Upv and Ipv with the proposed method.

The waveforms of the output voltage and current of three contrasted methods are showed in Fig. 6(b) (c), (d). As shown in Fig. 6(b), there is no restarting with the POC method, which eventually led to the global MPPT failure, because the power decrement caused by the irradiance variations in a MPPT period did not exceed the threshold power of the POC method. For the PSO method, if the power difference between the maximum power in memory and the current power exceeds the threshold power, it will restart the global scanning process, which led to frequently restarting and the dynamic efficiency loss. Fig. 6(d) shows that the proposed method track to the new global MPP just restarting one time, which mean that the proposed method avoids the problem of frequent restarting under dynamic case and the cumulative change of power and voltage is the reliable variables for judging environmental change.

\section{Conclusion}

The proposed global MPPT method in this paper is capable of tracking the global MPP under the static and dynamic environment. Its main advantages can be summarized as: 
- The method can track of the global MPP under the static and dynamic environment.

- The method is better than the existing method in the tracking speed under static environment, and without the steady oscillation.

- In the dynamic environment, this method has fewer restarting times, and the dynamic test efficiency is $4 \%$ higher than the existing method.

\section{Acknowledgements}

This work is supported in part by the National Natural Science Foundation of China under Project 51277051 and the Key Scientific and Technological Projects of Anhui Province -1201a0201004.

\section{References}

[1] Balasubramanian IR, Ganesan SI, Chilakapati N. Impact of partial shading on the output power of PV systems under partial shading conditions. IET Power Electronics, 2014; 7(3):657-666.

[2] Sera D, Mathe L, Kerekes T, Spataru SV, Teodorescu R. On the perturb-and-observe and incremental conductance MPPT methods for PV systems. IEEE Journal of Photovoltaics, 2013; 3(3):1070-1078.

[3] El-Dein MZS, Kazerani M, Salama MMA. An optimal total cross tied interconnection for reducing mismatch losses in photovoltaic arrays. IEEE Trans on Sustainable Energy, 2013; 4(1):99-107.

[4] Chen CW, Chen KH, Chen YM. Modeling and controller design of an autonomous PV module for DMPPT PV systems. IEEE Trans on Power Electronics, 2014; 29(9):4723-4732.

[5] Sharma P, Agarwal V. Maximum power extraction from a partially shaded PV array using shunt-series compensation. IEEE Journal of Photovoltaics, 2014; 4(4):1128-1137.

[6] Liu YH, Chen JH, Huang JW. A review of maximum power point tracking techniques for use in partially shaded conditions. Renewable and Sustainable Energy, 2015; 41(1):436-453.

[7] Kobayashi K, Takano I, Sawada Y. A study of a two stage maximum power point tracking control of a photovoltaic system under partially shaded insolation conditions. Solar Energy Material \& Solar Cells, 2006; 90(18):2975-2988.

[8] Ji YH, Jung DY, Kim JG, et al. A real maximum power point tracking method for mismatching compensation in PV array under partially shaded conditions. IEEE Trans on Power Electronics, 2011; 26(4):1001-1009.

[9] Alonso R, Ibaez P, Martinez V, et al. An innovative perturb, observe and check algorithm for partially shaded PV systems. In Proc. 13th European Conference on Power Electronics and Applications, Barcelona, Spain, 2009:1-8.

[10] Li SS, Zhang X, Zhang HK, et al. Global MPPT method based on power closed-loop control and PSO algorithm. Proceedings of the CSEE, 2014; 34(28):4809-4816.

[11] Koutroulis E, Blaabjerg F. A new technique for tracking the global maximum power point of PV arrays operating under partial-shading conditions. IEEE Journal of Photovoltaics, 2012; 2(2):184-190.

[12] Zhu YW, Shi XC, Dan YQ, et al. Application of PSO algorithm in global MPPT for PV array. Proceedings of the CSEE, 2012; 32(4):42-48.

[13] Lian KL, Jhang JH, Tian IS. A maximum power point tracking method based on perturb and observe combined with particle swarm optimization. IEEE Journal of Photovoltaics, 2014; 4(2):626-633.

[14] Ishaque K, Salam Z, Amjad M, et al. An improved particle swarm optimization (PSO)-based MPPT for PV with reduced steady-state oscillation. IEEE Trans on Power Electronics, 2012; 27(8):3627-3638. 